\title{
Scalability and scientific due diligence
}

\section{Neil Winterton}

Published online: 3 September 2011

(C) Springer-Verlag 2011

It was L. H. Baekeland who, in his 1916 Perkin Medal address to the Society of Chemical Industry in New York, said 'Commit your blunders on the small scale and make your profits on the large scale'. While he said this in the context of developing industrial chemical processes, its wider significance lies in the obvious but also possibly under-appreciated point, viz. the measure of success (whatever this might be) in any multi-step activity relies on the product of this measure for each individual step and not the sum. Also implicit in Baekeland's dictum is the importance of appreciating and anticipating scaling issues in technological development. While these are bread and butter matters for engineers, technologists and chemical companies engaged in such development, this is less true for academic chemists and others not directly involved in, or responsible for, the development process. Furthermore, when applied to global questions relating to systems for more sustainable energy, fuel and chemicals production, the issues of scale (and scalability) become even more complex and their wider appreciation yet more critical.

In a multi-step chemical synthesis the overall yield will be the product of the yields of the individual steps: a low yield for a single critical (and possibly overlooked) step will impact on the overall yield. In an industrial context, there is a need to decide whether to amend the synthetic route to avoid the low-yielding step; or, to research it in the hope of improving the yield through better understanding. An industrial operation (governed by the primary imperative of providing returns on investment) will usually opt, pragmatically, for the quicker less-costly option after

N. Winterton $(\bowtie)$

Department of Chemistry, University of Liverpool,

Liverpool L69 7ZD, UK

e-mail: N.Winterton@liverpool.ac.uk (if following Baekeland's advice) fully evaluating the pros and cons of the alternatives and applying expert judgement before coming to a decision.

A similar dilemma applies to the more complex problems found in the development and assessment of sustainable technologies, where there may be disagreement about the relative merits of research-led or a systemsdesign-led options (and even disagreement as to which the critical problematic step or steps might be). These are so-called 'wicked' (as opposed to 'tame') problems, multifaceted and multi-stakeholder, characterised by a lack of consensus on the goals to be aimed at, influenced by a range of scientific and other disciplines and subject to the flux of public opinion. With scarce resources and (in terms of the timescales of technological change) little time to identify more sustainable technologies how can we decide which are to be preferred: radical but disruptive technological approaches (such as the hydrogen economy) or seemingly more 'drop-in' alternatives (such as hydrocarbon production from biomass)? As it will not be possible to research and develop all the conceivable alternatives, back-of-the-envelope order-of-magnitude assessments are needed to rule out the obvious non-starters. Or, as Murray Gell-Mann said, 'it is vitally important that we supplement our specialized studies with serious attempts to take a crude look at the whole.'

However, sustainability-related research, development, impact assessment, promotion and comment by interestgroups and media now often proceed in parallel, seeking to influence as well as to inform (and too often it is difficult to distinguish between the two). The resulting confused and confusing environment makes difficult the development of an informed awareness of reliable evidence, a balanced assessment of its significance and the provision of a sound foundation for judgement and decision-making, whether by 
government, investors, experts, research funding agencies, activists or the wider public. Disconnects and confusion will be made worse if the discourse does not address factors, such as scalability and timescale, relevant to taking a laboratory discovery to the market place or to roll out globally an early-stage technology. Those making claims for some sustainability or environmental benefit for a piece of research in an academic publication or for some proposed research in a grant proposal should be required to 'take a crude look at the whole' and answer two simple questions: how far will it scale?; what are the prime constraints to scalability? Too many research papers play lipservice to these important factors. Too often, there is a tendency, particularly in the marketing of research results by journals and institutions, to accentuate potential benefits and to underplay, or even ignore, the negative aspects. False hopes can be raised and hares set running (with wasteful consequences in the disposition of research and development resources that might be more beneficially deployed elsewhere).

A 'crude look at the whole' does not necessarily require detailed expert analysis to provide a working estimate of a critical factor. For example, I have shown in a simple order-of-magnitude calculation with some defensible assumptions that land equal in area to the Netherlands would be required just for sheep-rearing to provide wool equivalent to the output of a single 500,000 $\mathrm{t} \mathrm{year}^{-1}$ poly(ethylene terephthalate) fibre plant.

Much research is currently focussed on the production of transportation fuels and chemical products from biomass. It may be tempting to argue that, because the quantity of solar energy intercepted by the Earth represents about 5,000 times humanity's current primary energy needs, a process to move to energy systems based wholly on its use should eventually yield to human ingenuity and investment, even taking into account the different efficiencies with which solar energy may be captured, converted, stored and moved about. While we are now only too aware of the extent of (and significance of) our dependency on fossil fuels, we may still have only a rather hazy notion, even in order-of-magnitude terms, of the precise quantity of oil and energy consumed globally each year. As a result, we may not easily be able to gauge whether, say, a new process for making a transportation fuel from biomass or some other source might enable fossil feedstock use to be reduced by a globally significant degree (its scale) or even whether some constraint might limit the extent to which it might be applied (its scalability). Giampietro and Mayumi, in their recent book, 'The Biofuel Delusion', provide an extreme example of non-scalability (and ignorance of scalability) associated with a report that the waste fat from liposuction procedures might be used as an energy source. As David MacKay says in his excellent book, 'Sustainable
Energy — without the hot air' 'Every little helps—a little!' In 'A Cubic Mile of Oil', Crane et al. express fossil and renewable energy sources in terms of 'cubic miles' of oil equivalent. Annual global oil use reached 1.06 mile $^{3}$ (or $4.42 \mathrm{~km}^{3}$ ) in 2006 , with the use of coal and natural gas, expressed in equivalent terms, 0.8 and 0.6 mile $^{3}$, respectively. On the same basis, the production of biodiesel and bioethanol combined and of PV electricity are equivalent to 0.0005 and 0.01 mile $^{3}$ of oil, respectively, in the same year. Sunlight captured annually as biomass (terrestrial and aquatic) is estimated to be equivalent to $20 \mathrm{mile}^{3}$ of oil. Meeting our needs from biomass rather than from oil might thus be viewed as a soluble technical problem, given sufficient time and effort, of harvesting and processing just $5 \%$ of the available plant material. Such thinking has spurred a major research and development effort into biofuel technology. However, it is now more generally known that things are more complicated because of (among other things) the non-equivalence of the energy-content per unit weight of oil and biomass, the greenhouse gas emissions associated, directly and indirectly, with fossil-fuel use in biomass growing, harvesting, transporting and processing, severe limitations of land- and water availability and the need (to provide the necessary labour for biomass production) to reverse the historic trend in migration from the countryside to cities. Biomass-based technology does not scale sufficiently to meet all our needs, suggesting a range of knotty social, economic and political challenges to be faced even greater than the technological hurdles to be overcome.

Each discipline or interest group will be aware of some of the parameters affecting scalability whose values may possibly be terminal for a technological opportunity (or at least may limit its applicability). Some of these parameters may be non-obvious to the non-specialist. Nor, such is the atomisation of academic disciplines, may their significance penetrate the less-than-permeable interfaces between disciplines or expert groups. Some, such as the importance of improved power densities for fuel-cell or battery developments for mobile applications, may be associated with technical challenges already the focus of specialist research. Others will have hardly penetrated into the general scientific consciousness but will be readily appreciated (such as the importance of using, identifying or developing plant strains for biomass production that minimise the loss of plant nutrients into harvested material). Yet others may represent potentially 'killer' vulnerabilities, or at least will give pause for further and deeper reflection to avoid premature enthusiasm or self-interested promotion of a research discovery through academic or corporate hype. Indeed, there will be constraints so widely known and generally accepted that it would be inexcusable for a piece of work to lay claim to a benefit that flew in the face of 
such information. While generalised claims for a contribution from research to a more sustainable technology are very easy to make, the significance that may be attached to them is such that they should be made with great care. Addressing the scalability question should, therefore, be a matter of scientific due diligence.

The need for rigorous and independent thinking, both broad and deep, allied to open-minded scepticism, has never been greater to prepare future generations (scientists and non-scientists) to navigate the difficult transition to sustainability. Unfortunately, these characteristics are insufficiently inculcated into our young students. In addition, for a variety of reasons, the space for teaching and rigorously testing the degree of understanding and recall of many basic chemistry fundamental ideas is under pressure from other 'sexier' more 'relevant' topics. Students are allowed to persuade themselves that facts can be obtained from the internet without having to commit them to memory; or that, as theories must be considered tentative and provisional, one theory should be considered as good as any other. All this gives rise to the impression that acquiring basic skills (particularly mental arithmetic and analytical thinking), incontrovertible facts and well-supported theories is educationally less important than making learning easier and attracting bored learners with more easily digestible curricula. While it is true that some learners do, indeed, require such methods to raise their educational attainment, it is my contention that this, when applied across the board, leads to an unfortunate proportion of school leavers being unable or unwilling, when moving to what should be the very much more rigorous intellectual environment of our colleges and universities, to see the importance of the linkage between course modules or an appreciation of the effort required to properly learn and understand. Failure of learning is, increasingly, seen as a failure of teaching. When a leading UK university changes the name of its 'Teaching and Learning Committee' to the 'Student Experience Committee' one can see how far the rot has gone! Teachers must not avoid things that are too hard as, if these things are not taught, then they will be deemed by students to be unimportant.

Students need to be encouraged to develop the mental abilities necessary for testing whether a calculator-derived answer looks reasonable as well as for scoping out problems as a precursor, for instance, to designing a programme of work. The identification of critical variables that affect the value of a poorly-defined parameter to produce workable estimates from less-than-complete data has become known as Fermi calculations after Enrico Fermi who expected his students to develop and to use them. These are just the methods needed to assess scalability and are of wider, more general, utility. There is a good case to be made that science and general curricula, throughout our educational systems, are in need of wider reform to make them fit to develop similar capabilities in the next generation of graduates so they can meet the challenges of sustainability. The conventional mindset, conditioned over the last century of cheap oil and energy and which influences the content and focus of teaching and research content particularly of chemistry and related disciplines, also needs to be challenged. Indeed, one can ask whether the decline in the attention given to teaching the laws of thermodynamics and their significance and application has been the unintended consequence of this mindset. Energetics should now become an increasingly significant component of our chemistry courses. There is even a case for teaching basic thermodynamics to the very young. We also need to value the contribution that the generalist may make alongside that of the specialist. It took me too long to become aware of the books of people like Vaclav Smil who provides useful insights and associated data on many contemporary sustainability-and energy-related questions.

There is a third aspect of Baekeland's dictum that is worth addressing in a modern context. Reliable and robust decision-making requires the decision-maker to be informed sufficiently to exercise reasonable judgement. However, there is a paradox, viz. no one individual can take in all the necessary information relevant to sustainability; on the other hand, individuals need to be aware of all factors relevant to a problem to aid the development of their own well-founded perspectives. In an industrial operation there is a hierarchy of power with associated lines of communication and responsibility that supports decision making. In a social and political context, however, we prefer the decision-maker to be democratically accountable, experience having shown that centralising unaccountable power into the hands of an individual or a ruling elite can have serious consequences. On the other hand, democratic politics does not lend itself to efficient or well-founded decision making. Efforts to improve this with ever more refined means of representation are doomed to failure if only because of the operation of Arrow's Impossibility Theorem which states that collective decision-making, if logical, cannot be completely fair and, if fair, will not be logical. As Winston Churchill famously said 'Democracy is the worst form of government, except for all the others.' This is still true despite the role played by the $24 \mathrm{~h}$ 'news' industry and global and instant mass communications. Instant reaction, with rapid developing political pressure, tends to lead to short-term responses based on less-than-fully-informed comment rather than on fully-considered information. Knowledge and experience tend now to be ignored and or its importance marginalised. Capturing the headlines with some bold opinion without all the facts, or without the insight and experience to analyse and to understand, can now be shared with very many, and 
can be manipulated by some. Of course, this in some respects is not new: the ancient Greeks knew a thing or two about the power of rhetoric as a means of influencing opinion. The difference in their case is that such influence was exercised verbally and directly to those who would have to decide and was not first selected and mediated by individuals and groups with vested interests. We do have to live with what we have and this means working to improve things where we can, including our education systems so that the next and future generations are able to deal effectively with the global legacies they are being bequeathed. I have a possibly romantic view that there exists (under the consumerist and celebrity-obsessed froth) a collective common sense that arises from general and everyday experience and interaction with the world around us. However, common sense can take us only so far. Scientists and others need, therefore, to stay true to the scientific ethic in searching for essential and sometimes inconvenient truths and, recalling Baekeland's words, providing knowledge and insight to ensure our political systems avoid making too many big mistakes in our search for practical and scalable sustainable technologies. However, gaining support in a democracy for uncomfortable change will also require unprecedented altruism, vision and forbearance. I have quoted Freudenburg and Kates before: the need for 'a deeper kind of prudence' and 'a capacity to worry intelligently' is as great as ever. 